\title{
New Endeavors of (Micro)Tissue Engineering: Cells Tissues Organs on-Chip and Communication Thereof
}

\author{
Haysam M.M.A.M. Ahmed ${ }^{a} \quad$ Liliana S. Moreira Teixeira ${ }^{a}$ b \\ aDepartment of Developmental Bioengineering, Technical Medical Centre, University of Twente, \\ Enschede, The Netherlands; ${ }^{b}$ Department of Clinical Sciences, Faculty of Veterinary Medicine, Utrecht University, \\ Utrecht, The Netherlands
}

\section{Keywords}

(Multi)organs-on-chips · Tissue engineering · In vitro models · Precision medicine

\begin{abstract}
The development of new therapies is tremendously hampered by the insufficient availability of human model systems suitable for preclinical research on disease target identification, drug efficacy, and toxicity. Thus, drug failures in clinical trials are too common and too costly. Animal models or standard 2D in vitro tissue cultures, regardless of whether they are human based, are regularly not representative of specific human responses. Approaching near human tissues and organs test systems is the key goal of organs-on-chips $(\mathrm{O} o \mathrm{C})$ technology. This technology is currently showing its potential to reduce both drug development costs and timeto-market, while critically lessening animal testing. $\mathrm{OoC}$ are based on human (stem) cells, potentially derived from healthy or disease-affected patients, thereby amenable to personalized therapy development. It is noteworthy that the OoC market potential goes beyond pharma, with the possibility to test cosmetics, food additives, or environmental contaminants. This (micro)tissue engineering-based tech-
\end{abstract} (developmental) biology, (bio)materials, microfluidics, sensors, and imaging. The enormous potential of $\mathrm{OoC}$ is currently facing an exciting new challenge: emulating crosscommunication between tissues and organs, to simulate more complex systemic responses, such as in cancer, or restricted to confined environments, as occurs in osteoarthritis. This review describes key examples of multiorgan/tissueon-chip approaches, or linked organs/tissues-on-chip, focusing on challenges and promising new avenues of this advanced model system. Additionally, major emphasis is given to the translation of established tissue engineering approaches, bottom up and top down, towards the development of more complex, robust, and representative (multi) organ/tissue-on-chip approaches.

(c) 2021 S. Karger AG, Basel

\section{Introduction}

Preclinical models, both in vitro and non-human in vivo, play a fundamental role in drug development. However, it has now become increasingly evident that standard steps involved in drug development are facing crit-

Correspondence to:

Liliana S. Moreira Teixeira, l.s.moreirateixeira@utwente.nl 
ical challenges, mainly due to drug failure in clinical trials, after having passed preclinical studies. As a result, not only are costs increased, but also the development of novel therapies is hindered. At the preclinical stage, in vitro cell culture models, typically restricted to 2 -dimensions (2D), are the gold standard for initial screening of activity and specificity of drugs. Despite having played a crucial role in advancing the drug development process, these simple models are, thus, fairly limited due to incapacity to mimic the complexity of native tissues or organs, more specifically, the absence of a native-like physicochemical 3D microenvironment, vascularization, fluid flow, shear stress, and also importantly, a multiorgan context. In fact, 2D model-based pharmacokinetics often fail to accurately predict ADMET (absorption, distribution, metabolism, and excretion and toxicity) of a drug at the biological target, leading to inaccurate dosages [Duval et al., 2017]. To circumvent the shortcomings of 2D cell cultures, $3 \mathrm{D}$ culture models have emerged as a viable alternative due to the improved tissue organization and prolonged maintenance of cell functions [Pampaloni et al., 2007]. However, standard 3D culture models also tend to fall short on faithfully reproducing the characteristics of living organs, including tissue-tissue interaction, shear stress provided by fluid flow, gradients of oxygen and cytokines, and/or mechanically active microenvironments [Huh et al., 2011a]. On the other hand, in vivo preclinical investigations, which rely on using animal (mostly rodent) models, are still regarded as a key step in drug development, since they maintain the significant organ interactions taking place in complex living systems. Animal models allow the evaluation of organ-organ cross-talk, and more faithfully reproduce pharmacokinetic and toxicological responses, as compared to standard 2D and 3D systems. However, these models are not without limitations. For example, the interspecies discrepancies between laboratory animals and humans hamper the transferability of the results, or extrapolation of effects and responses to their human counterparts [Sung et al., 2014b; Everitt, 2015]. Therefore, it is increasingly recognized by the scientific community and regulatory entities that preclinical assessments based on animal models often lead to poor-efficacy prediction [Saeidnia et al., 2015]. Markedly, failure estimate rates can even reach $90 \%$ [Pound and Ritskes-Hoitinga, 2018]. Consequently, advancements in the models available are undeniably urgent.

Biological advances, including harnessing the potential to steer primary and stem cell-derived human cells, combined with novel biomimetic materials, and break- throughs in bio- and microfabrication approaches, have opened up new avenues for preclinical models. These multidisciplinary advances result in unprecedented in vitro recapitulation of key functionality, structural organization, and interaction dynamics of human tissues and organs. In this context, the field of organs-on-chips $(\mathrm{OoC})$ is flourishing with increasing recognition of the potential to bridge the gap between in vitro/in vivo validation and clinical translation, with the promise to not only improve the predictive value of preclinical studies dearly needed to advance drug development, but also significantly contribute to the unravelling of disease mechanisms and enable precision medicine approaches [Low et al., 2021a].

The rapid pace of OoC translational opportunities are paving the way to wide-ranging academic, medical, and commercial avenues. For instance, recent publications have highlighted the impact of $\mathrm{OoC}$ in critical diseases, such as cancer, and/or toxicity screening, which include relevant organs such as liver, heart, lung, gut, and kidney, reflecting the high impact of this novel technology in clinical applications [Ronaldson-Bouchard and Vunjak-Novakovic, 2018]. In parallel, from the industrial application point of view, the significance of involving stakeholders in early assessment, from academic to regulatory entities (e.g., FDA and EMA) to product development specialists, is undeniably necessary, and have been extensively analyzed, including potential challenges over the path of both translation and commercialization. These topics have been discussed by other authors [Middelkamp et al., 2016; Watson et al., 2017; Low et al., 2020].

In this review, we aim to discuss how in vitro preclinical models, ranging from organoids to microfluidic OoC, can be developed to improve and accelerate preclinical studies, thus boosting drug development and/or supporting a deeper understanding of human pathophysiology. We will describe the steps undertaken towards establishing multi-OoC models, which are undeniably more representative of human systemic-like physiology, and hence clinically more relevant as preclinical models for studying both pathophysiology and complex pharmacological responses. Following this, we will discuss strategies to facilitate the use and commercialization of OoC, from design considerations, to adaptations, to stakeholder demands. Finally, we will also focus on the advantages, bottlenecks, and potential for further exploring these sophisticated representations of human tissues and tissue systems models in uncharted areas or applications. 
Fig. 1. In vitro and in vivo models: from $2 \mathrm{D}$ to $3 \mathrm{D}$ dynamic microfluidic OoC. Comparison between complexity levels of assets or characteristics of the various model types. The relative levels of complexity of the different selected models portray compiled data and overviews from the different models [Huh et al., 2011b; Marx, 2012; Bhise et al., 2014; Halldorsson et al., 2015]. Overall, OoC show high potential to expand the capabilities of the conventional in vitro and in vivo models by enabling highprecision, high-throughput, and decreasing costs due to implemented automation, while maintaining a physiologically relevant humanized context.

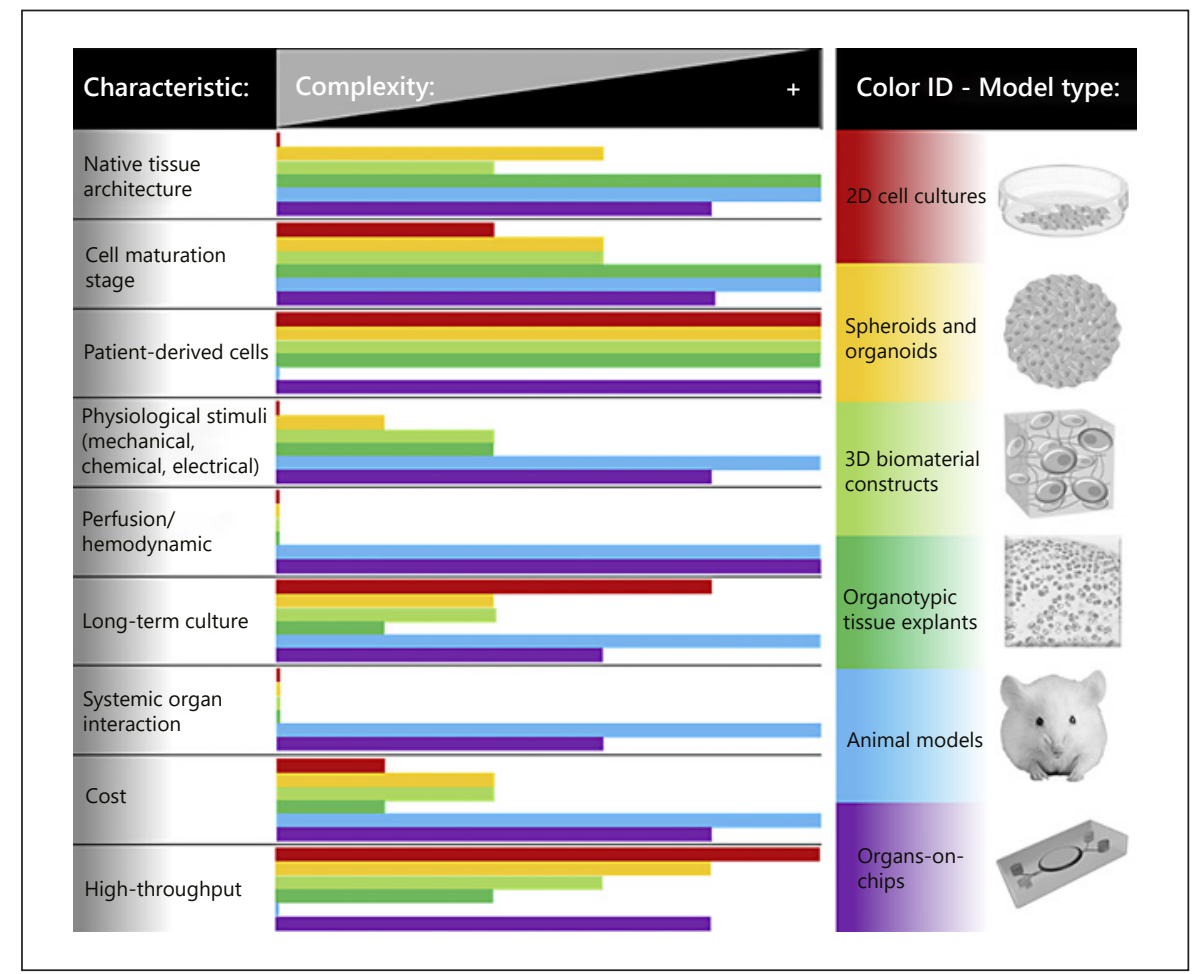

The Evolution of Advanced 3D in vitro Models: From Spheroids to Microfluidic OoC

Although the description of spheroids and organoids is beyond the scope of the present review, we ought to emphasize that these culture systems present undeniable advantages over typical 2D systems. Namely, spheroids and organoids are able to maintain more complex cellular functions and interactions, which renders these $3 \mathrm{D}$ cell culture approaches greatly useful for high-throughput screening for drug development, for example as toxicology testing models, and/or as disease models. Alternatively, 3D biomaterial construct models also present several advantages as advanced models, as these are composed by synthetic or naturally derived biomaterials/polymers, closely simulating cell-ECM interactions. Patient-derived tissue explants are also commonly used as physiologically representative $3 \mathrm{D}$ ex vivo models, as these are relatively easy to obtain and inexpensive to maintain. Despite being highly informative, the abovementioned models still often fail to emulate both the structural and dynamic complexity of tissues and organs, subsequently resulting in incomplete or even inadequate tissue/organ responses. Overcoming these limitations, OoC platforms stand out due to their advantageous combination of defining characteristics: (a) mimicry of na-

Cells Tissues Organs on-Chip tive architecture of tissues, closely replicating their key $3 \mathrm{D}$ nature and organization; (b) enabling the integration of multiple cell types (e.g., parenchymal, stromal, vascular, and immune cells), thereby reflecting more physiologically relevant cell-cell/cell-tissue interactions; and, (c) incorporation of engineered stimuli, such as biomechanical forces (e.g., stretch/strain forces for actuated tissues, or hemodynamic shear forces for vascular tissues), electrical cues, and biochemical gradients relevant to the tissue being modelled, as shown in Figure 1. One of the very first OoC models, the "lung-on-a-chip," published in 2010 [Huh et al., 2010a], set the stage for these promising engineered microdevices, ultimately designed to mimic key features of organ structures, functions in both healthy and disease conditions, and their reaction to stressors and pharmaceutical compounds. A decade later, OoC models have shown great progress due to major advances in the microfabrication technologies, sensors, imaging, and (systems) biology, uniquely delivering a balance between fidelity and feasibility, whilst maintaining robustness and throughput.

\section{D Biomaterial Constructs and Organotypic Tissue Explants}

The (bio)chemical and mechanical properties of the biomaterials, combined with the physical 3D support, 
provide cues to steer cellular behavior, such as stiffness, porosity, surface chemistry, biodegradability, and cell compatibility. A myriad of biomaterials have been used to engineer 3D cell cultures, ranging from the broadly used collagen coatings or Matrigel, to more complex in situ cross-linkable bioinks for 3D bioprinting (as reviewed in more detail in Moroni et al. [2018] and Suvarnapathaki et al. [2019]). However, the main concerns in using 3D biomaterial construct models include: (1) finding the most suitable material among the wide range of biomaterials available for a specific application can be time consuming, and (2) concerning natural source materials, it may be a challenge to control matrix properties and assure reproducibility, derived from inherent batch-to-batch variability. Synthetic polymers, on the other hand, circumvent the reproducibility problem faced with natural hydrogels. One of the most widely used synthetic polymers is gelatin methacryloyl (GelMA) due to its tunable mechanical and biological properties, biocompatibility, and the versatility of its application for drug delivery, 3D printing, and tissue engineering [Yue et al., 2015; Zhu et al., 2019].

Conversely, in tissue explants derived from primary tissue from patients, the cells are preserved in their native extracellular matrix, which renders this model easy to produce and inexpensive. Similar to 3D biomaterial constructs, this type of model also allows growth factor and cytokine stimulation, implementation of physical injury, and is compatible with osmotic and/or mechanical loading regimens [Bush et al., 2005; Brown et al., 2015; Johnson et al., 2016]. Yet this organotypic model also presents a few inherent disadvantages, mostly related to the limitation of primary tissue available from the same source, high variability due to donor variation, induced cell death at the explanted tissue's edges, possible need to include support surrounding tissues to retain viability and functionality, and, lastly, native tissue characteristics can suffer alteration in static ex vivo culture environments. There are currently no regulations regarding the biomaterials to be used in in vitro models. However, regulations for biomaterials to be used in medical devices and clinical applications (e.g., implants) might be useful for OoC manufacturers to take into consideration when designing their in vitro models.

\section{Spheroids and Organoids}

Spheroids consist of spherical cell aggregates, with a certain degree of self-organizing and self-renewing abilities, which can be composed of single-cell suspensions derived from cell lines or patient-derived primary cells, typically cultured in ultra-low attachment platforms. Co- culture approaches are also used to better mimic more complex native microenvironments, by addition of endothelial, mesenchymal, and/or immune cells, for example. Although undoubtedly valuable as 3D model systems for several applications, spheroids display limited tissue-like structure, self-renewal, and differentiation potential.

Organoids are able to overcome withstanding limitations of spheroid model systems by providing a higher level of complexity [Mead and Karp, 2019]. Organoids typically originate from tissue-derived stem cells or human-induced pluripotent stem cells (hiPSCs), cultured within an ECM-like hydrogel matrix, that allows structural changes and cell-cell interactions, which, in turn, form organized structures resembling the in vivo tissue equivalents [Lancaster and Knoblich, 2014]. Thus, this culture model displays cell compositions and structure more similar to native tissues. Moreover, as this model originates from self-renewing stem cells able to differentiate into cells of all major cell lineages, it has the capacity to display an intrinsic ability to self-organize and recapitulate developmental programs. Such features render this culture system with a higher biological relevance, amenable to niche manipulation and gene sequencing. However, this advanced in vitro system may tend to be retained at a fetal level of maturation, which, in turn, is highly dependent on the supplied organoid's microenvironmental cues. As a representative example, intestinal organoids have demonstrated to be an excellent model to assess underlying molecular mechanisms and functional gastrointestinal processes [Sato et al., 2011; Dekkers et al., 2013]. In this model, the polarization of enterocytes of the intestinal organoid was successful, which enabled the assessment of nutrient transport and sensing. Another case of effective utilization of this type of model is kidney organoids to study kidney diseases and regeneration [Takasato et al., 2015]. Interestingly, hiPSCs can be generated from patients with inherited kidney disease, or, by genome editing of healthy patient-derived hiPSCs, mutations of target genes can be introduced via CRISPR/Cas9. This approach can ultimately enable drug screening to find new therapeutic approaches and the analysis of mechanisms of disease due to the mutation. Recently, organoid technology has also been demonstrated to be a reliable 3D model of cancer behavior during disease progression, for numerous cancer types such as pancreas, prostate, ovary, and colon [Matano et al., 2015; Baker et al., 2016; Puca et al., 2018; Kopper et al., 2019]. This approach is an important step towards personalized medicine, as it allows the in vitro evaluation of therapy responses, single or even combined, namely chemo-, ra- 


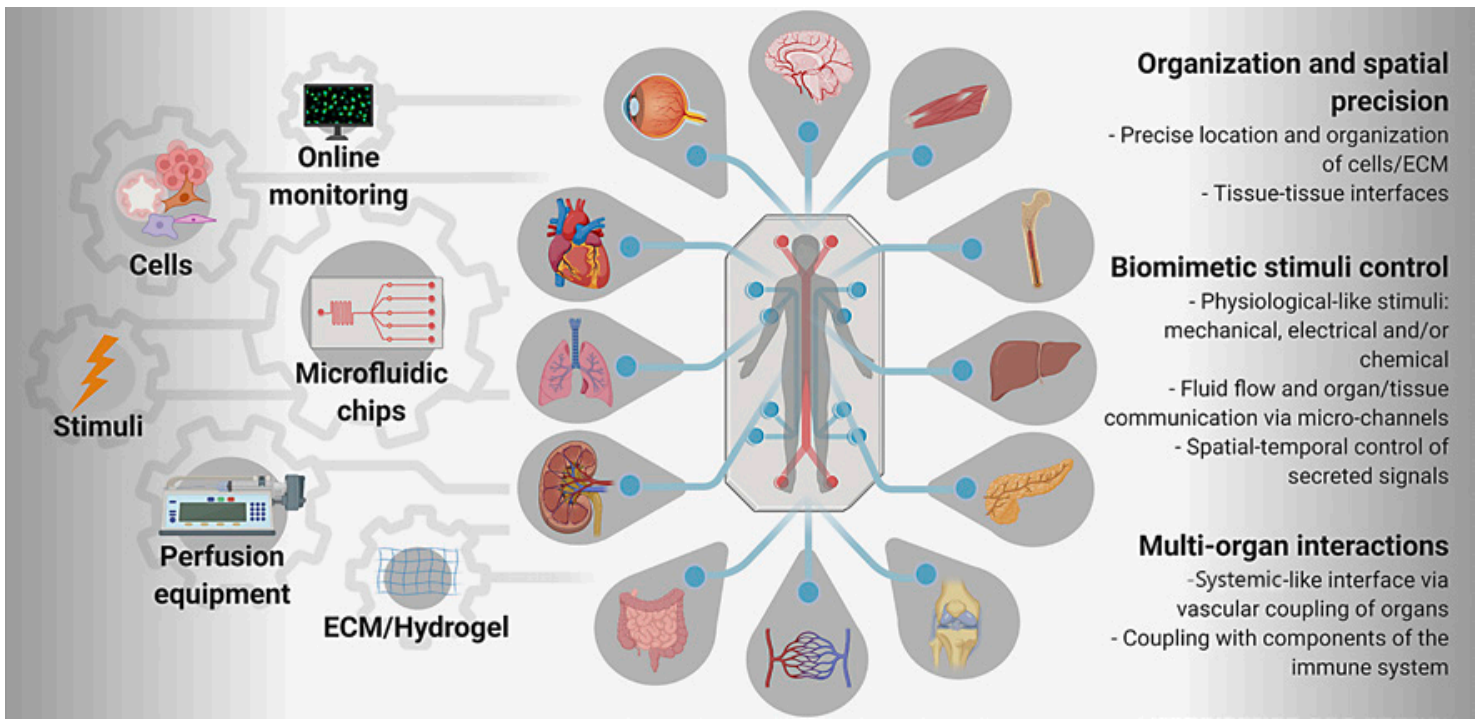

Fig. 2. Concept representation of an OoC device: key considerations for design and fabrication (left side); advantages of OoC (right side), highlighting the unique features of this advanced model, expected to play a pivotal role in streamlining the path towards clinical trials. Created with BioRender.com.

dio-, and/or immunotherapy. Similar to other organoid models, CRISPR/Cas9 technology has also recently been applied for genome engineering of cancer organoids [Driehuis and Clevers, 2017; Takeda et al., 2019; Artegiani et al., 2020].

\section{Organs-on-Chips}

The development and validation of $\mathrm{OoC}$ as preclinical models has gained increasing attention and recognition over the last decade, with the promise to raise efficacy, safety, and, ultimately, hasten drug discovery, instigating outstanding interest in areas such as pharmacology and toxicology [Low et al., 2021a]. Indeed, these models are playing an increasingly prominent role in the enlightenment of complex human diseases where representative models are still lacking. Although many of the recent insights achieved with OoC would not be possible using animal models, it is fundamental to perceive these advanced platforms as complementary models, contributing to reducing the number of experimental animals, instead of complete replacements of the existing ones. In fact, established animal models are also fundamental for the full validation of OoC platforms. Notably, OoC models are not designed to mimic the functions of entire organs. Instead, these advanced platforms are targeted at replicating human-based, physiologically relevant, 3D cross-sections of key functional subunits of organs or tissues. Thus, OoC models are typically devised with focus on specific questions, recreating the minimal organ/tissue unit and the minimal interactions necessary to replicate in vivo functionality.

\section{Technological Advancements}

OoC technology benefited greatly from decades of research in the field of microfluidics [Domachuk et al., 2010; Barata et al., 2016; Du et al., 2016; Convery et al., 2019]. Although originally developed for the electronics industry, $\mathrm{OoC}$ is conceivably one of the most rapidly expanding research areas of microfluidics at the moment. Uniquely combining physiologically relevant structural units, namely cellular microenvironments and 3D structures, together with dynamic cues, or physiological forces, OoC models offer the potential for unrestricted imitation of crucial tissue-tissue responses, as shown in Figure 2 . The physiological-like perfusion, typically within multicompartmental channels, delivers the 3D microengineered cell culture environments the required dynamic cues to better mimic in vivo cell-cell interactions, tissues, and organs. With the distinct ability to combine perfusion with kinetic modelling, various toxicokinetic processes can be reproduced, namely absorption, metabolism, and/or debris removal. Chamber and channel compartmentalization not only allows fluid or air flow, but also permits pressure-driven dynamic mechanical cues, as shear stress and/or cyclic mechanical strain, achieved by pressure-controlled fluid flow rates and channel ge- 
ometry, or stretching/compressing elastic membranes, respectively [Park et al., 2009; Huh et al., 2010b; Kim et al., 2012]. Compartmentalization of microfluidic channels has also been developed to enable concentration gradient generation on OoC platforms [Wang et al., 2017]. The obtained microfluidic gradients show high predictability and reproducibility, whilst maintaining real-time control of perfused solutions and easy quantification readouts (e.g., fluorescently labelled solutions quantifiable by microscopy; incorporation of sensors for continuous assessment). Thus, several approaches for integrated gradient-based microfluidic devices have been successfully developed for high-throughput drug screening, which enabled correlations between higher intracellular drug concentration and increased cytotoxicity, for example [Gao et al., 2012; Carvalho et al., 2019].

\section{Increasing Biological Complexity}

The above-described advanced in vitro models, namely spheroids, organoids [Park et al., 2019; Low et al., 2021a], 3D biomaterial constructs [Bahmaee et al., 2020], and organotypic tissue explants [Brooks et al., 2017], can be seamlessly combined with OoC platforms, which highlights the flexibility of this advanced technological tool. Also, when combined with hiPSC technology, individualized target tissues can be differentiated, prior or within on-chip platforms, circumventing the possible cell source limitation. This type of combined models holds the promise to advance personalized medicine approaches, since specific genetic information can be carried. Recently reported examples include the formation of a complex human retinal model integrating seven different cell types through the vascularization of retinal organoids in a microfluidic chip [Achberger et al., 2019], and the creation of a heart-on-chip [Zhang et al., 2016]. The latter elegantly combines bioprinting of an endothelialized myocardium with a $3 \mathrm{D}$ construct. Additional extensive descriptions on the development of $\mathrm{OoC}$, as well as the various methodologies describing its use in combination with stem cells, have already been discussed in other reviews [Ertl et al., 2014; Geraili et al., 2018; Rothbauer et al., 2018; Cochrane et al., 2019; Nawroth et al., 2019].

\section{Towards Multi-OoC Models for Drug Development and Disease Modelling}

Various organs have been studied and key aspects were replicated on-chip, including lungs, liver, kidney, intestines, and tumor microenvironments [Benam et al.,
2016; Shim et al., 2017; Carvalho et al., 2019; Sateesh et al., 2019; Langerak et al., 2020; Moradi et al., 2020]. However, these models are largely based on single organs, which fail to recapitulate the physiological complexity of the native in vivo situation. Therefore, in recent years, the integration of multiple organ functions on-chip has gained increasing attention. Linkage of multiorgan/tissue systems is crucial to more accurately model complex organ-organ/tissue-tissue interactions towards applications in, for example, pharmacokinetic profiling, drug screening, and systemic disease modelling [Sung et al., 2014a; An et al., 2016].

\section{Multi-OoC Models for Drug Development}

Multi-OoC integrated platforms are currently being developed and validated for systemic-like drug screening and/or evaluation of side effects, as well as for complex disease modelling, where replicating organ-organ/tissuetissue communication is imperative. In fact, combined academic, governmental, and industrial efforts to develop a "body-on- chip," recently led to the development of ten linked OoC [Edington et al., 2018]. By combining the multi-OoC biological data with computational modelling, a predictive systemic-like pharmacokinetic profile was achieved and validated [Edington et al., 2018], as shown Figure 3a. Another representative example of a four-organ-chip system established for ADME profiling is shown in Figure 3b. In this study, a microphysiological system maintaining the long-term functionality of four organs: skin, intestine, liver, and kidney, has been successfully established [Maschmeyer et al., 2015a]. Another highly relevant example consists of four connected OoC platforms, developed to evaluate five different compounds, with pre-identified side effects [Oleaga et al., 2016]. Similarly, in another study, individual OoC were connected via an endothelial layer, assuring linked perfusion, while maintaining a separate compartment for each OoC, with tissue-specific media, as shown in Figure 3c [Ronaldson-Bouchard and Vunjak-Novakovic, 2018]. In another study, the cardiotoxicity of cyclophosphamide and terfenadine were accurately modelled using a liverheart-on-a-chip, showing clear advantages over heartonly models [Oleaga et al., 2018]. Similarly, a liver-kidney model revealed novel mechanisms of aristolochic acid nephrotoxicity [Chang et al., 2017]. Application of multiOoC can also be extended to study reprotoxicity. As a representative example, an $\mathrm{OoC}$ model of the female reproductive tract, including ovarian tissue, fallopian tube, uterus, and cervix modules, was connected to a liver module to assess reproductive toxicology (Fig. 3d) [Xiao et al., 


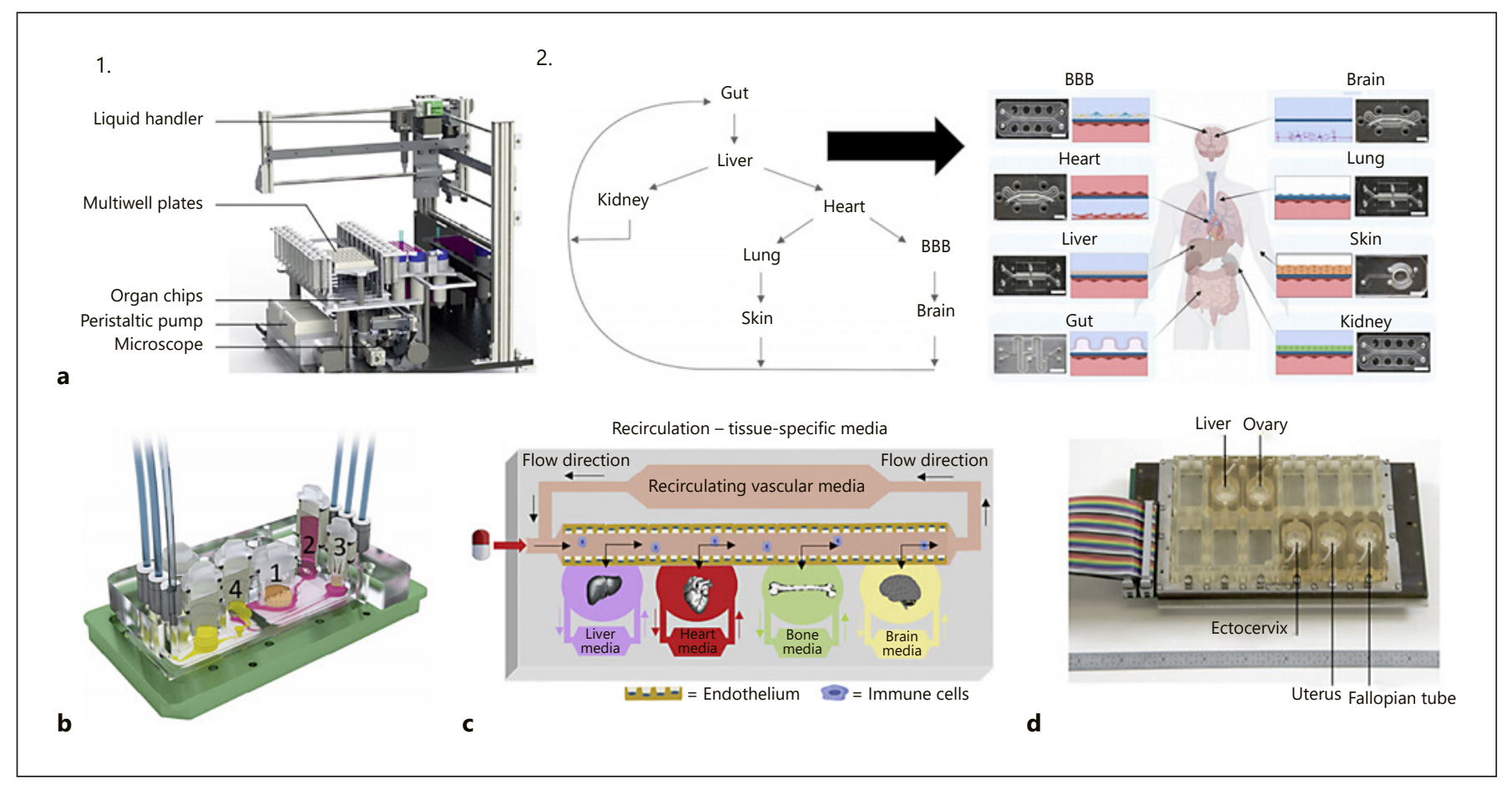

Fig. 3. Selected examples of features and platform designs for multi-OoC platforms. a 1. Overview of the "Interrogator Device," which enabled the connection of eight OoC, achieved by programed unit sampling and interconnected perfusion; 2. Linking scheme of eight vascularized, two-channel OoC, successfully connected and cultured for 3 weeks. This multi-OoC setup comprised the following units: intestine, liver, kidney, heart, lung, skin, bloodbrain barrier (BBB), and brain. Reproduced from Novak et al. [2020], with permission from Springer Nature. b 3D view of the microfluidic four-OoC device, which comprises tissue culture compartments for intestine (1), liver (2), skin (3), and kidney (4). Reproduced from Maschmeyer et al., [2015b], with permission from the Royal Society of Chemistry. c Development of individual
OoC connected to a selective membrane barrier (endothelial layer), enabling OoC the integration with recirculating media perfusion that connects all $\mathrm{OoC}$, while preserving the tissue-specific media composition for each OoC. The recirculating media includes biomimetic components (e.g., circulating immune cells). Reproduced with permission from Ronaldson-Bouchard and VunjakNovakovic [2018]. d A microfluidic culture model of the human reproductive tract and 28-day menstrual cycle, simulating the in vivo female reproductive tract and the endocrine profile. The following organ modules were included: ovary, fallopian tube, uterus, cervix, and liver. Reproduced from Xiao et al. [2017], with permission from Springer Nature.
2017]. In another recently reported platform, testicular organoids were coupled to liver coculture spheroids to study the reprotoxicity of an antineoplastic drug, with known germline effects [Baert et al., 2020]. Interestingly, some of these systems also incorporate a variety of biosensors for real-time noninvasive readouts, to support long-term cell culture and recurring drug testing.

\section{Multi-OoC as Complex Disease Models and Targeted Therapy Screening Platforms}

Disease modelling on OoC holds the promise of playing a pivotal role in the development of precision medicine. While initially many OoC models were conceived based on cell lines, current models tend to be based solely on patient-derived primary or hiPSCs, enabling the ad- dition of the patient's genetic information. The "patienton-a-chip" models allow: (a) patient stratification, by identifying subpopulations that react optimally to specific therapies, dosages, or combined treatments; and, (b) development of patient-specific platforms to model patients with rare diseases, for example. Such specific platforms not only enable comparison and treatment optimization, but additionally contribute to understanding the mechanistic effects of disease progression or effect of drugs in specific pathways, thereby, paving the way to the use of OoC for precision medicine. Many examples of the application of OoC for precision medicine have focused on tumors [Jeong et al., 2016; Hassell et al., 2017; Lee et al., 2018; Miller et al., 2018], with particular importance placed on establishing multi-OoC when applying this 
technology to cancer metastasis [Liu et al., 2019]. Other relevant multiorgan systems include steatosis [Lee and Sung, 2018] and inflammatory bowel syndrome [Trapecar et al., 2020]. With this approach, the mechanism of disease progression was investigated via multiomics, ultimately highlighting the important interactions between tissue homeostasis, immunity, and metabolism.

\section{Potential Bottlenecks and Challenges}

\section{Design and Fabrication Specifications}

Designing OoC platforms typically starts by defining the channel diameters and length, culture chamber dimensions, potential presence of membranes or support ECM-like scaffolds, and placement of inlets/outlets, which can influence the flow rate, and, therefore, the shear stress exerted on the tissues [Stone et al., 2004]. To better mimic the in vivo microenvironment, some tissues require biomechanical stimuli, such as stretch forces for lung alveolar tissues [Kaarj and Yoon, 2019], shear stress forces for vascular tissues [Ting et al., 2013; Gnecco et al., 2019], or compressive forces for, for example, bone and articular cartilage [Park et al., 2012; Occhetta et al., 2019]. The first developed lung-on-a-chip platforms already included mechanical actuation on-chip via lateral vacuum chambers, adjacent to the perfusable cell culture chambers [Huh et al., 2010a]. Imitation of the biomechanical forces associated with breathing was achieved by the periodic application of vacuum. In turn, these biomimetic movements induced stretching/relaxation of the membrane horizontally placed between the culture chambers, onto which cells were attached. Biomechanical forces are also important to accurately recapitulate the mechanical factors involved in e.g., osteoarthritis pathogenesis or inducement of this disease model [Gottardi, 2019]. Other key features may be integrated during chip conceptualization, namely the optical properties of the materials used. For example, heart-on-chip platforms benefit from being fabricated from optically clear materials, to allow direct imaging of cardiac contractions [Zhao et al., 2020]. Design considerations related to sensor incorporation are fundamental for applications in neural or muscular platforms, for example, which typically incorporate (multi) electrode arrays [Maoz et al., 2017]. Besides chip design specifications for each tissue or organ, prevention of bubble formation may also need to be addressed, as entrapped bubbles can obstruct medium perfusion [Lochovsky et al., 2012]. The presence of bubbles within these types of microsystems not only alter fluid flow profiles, affecting shear rates, but can also critically compromise uniform cell seeding or even viability. Incorporating bubble traps can be an easy solution to integrate in OoC design and fabrication, to avoid trouble-shooting at later stages.

Besides conceptualization and design features to be addressed during the development of $\mathrm{OoC}$, the materials used for chip fabrication or surface coating are also highly relevant, as these can affect cell attachment, gas permeation, as well as the absorption/retention of small molecules. Conventionally, OoC models have been fabricated using polydimethylsiloxane (PDMS) elastomer. Typically, chip architecture is created by soft lithography, enabling the imprint of internal structures, namely the microscale fluid channels, cell culture chambers, and actuation units. PDMS-based chips render a degree of design flexibility, due to the possibility of tuning their elastic properties to a certain extent, thereby enabling the controlled execution of biomechanical forces, as well as their low manufacturing cost. PDMS also enables optically clear platforms that allow real-time tissue imaging and online integration of (multiple) sensors [Zhang et al., 2017a]. Furthermore, PDMS chips can be further modified via micro- and nanopatterning to modulate cell behavior using resins, hydrogels, or ECM components, as previously described by others [Baker et al., 2013; Barata et al., 2017; Nouri-Goushki et al., 2019]. Nevertheless, PDMS-based models suffer from some drawbacks, namely the requirement of specialized equipment (e.g., cleanroom facilities), potential absorption of small molecules (e.g., retention of drugs), and some restrictions related to replicating some tissues' microarchitecture [Toepke and Beebe, 2006; Knowlton et al., 2016]. Alternative materials for chip fabrication include glass, thermoplastics, and resins. Material selection for chip fabrication is achieved by obtaining a workable compromise between material availability, topography requirements, speed of fabrication, required skills/equipment/facilities, and affordability. Testing the adsorption, absorption, and cyto-compatibility for each material used is highly recommended at early design and fabrication stages [Regehr et al., 2009; Zambito et al., 2020].

Recently, 3D bioprinting emerged as a promising advanced technology, highly compatible with OoC fabrication. Thus, 3D bioprinting has assisted in the development of the next generation of OoC systems due to: (a) processing versatility; (b) rapid generation of microchannels and culture chambers with high shape fidelity; (c) user-friendly equipment; and, (d) compatibility with bioinks, bioactive molecules, and multiple cell sources. This combination of advanced technologies undoubtedly ac- 
celerates the manufacture of more structurally complex and biomimetic 3D tissue models [Waheed et al., 2016; Park et al., 2018]. 3D bioprinting methods, including stereolithography, melt-electrowriting, and extrusion-based printing, enable the biofabrication of intricate 3D (micro) structures. These methods allow precise control of the spatial distribution of supportive biomaterials, potentially in combination with various cell types and ECM, typically assisted by CAD (computer-aided design) models [Mandrycky et al., 2016; Kankala et al., 2018]. Altogether, $3 \mathrm{D}$ bioprinting technology combined with OoC contributes tremendously to rapid and continuous model generation, facilitating the emulation of intricate (patho) physiological features with high precision, fabrication speed, and low cost [Low et al., 2021b].

\section{Biological Factors}

When developing OoC platforms it may be more beneficial to engineer simple tissues that can model key aspects or functionality of whole tissues or organs. By also taking into account the inherent interaction between organs or tissues, these platforms may capably provide relevant and effective answers for the questions proposed. Important considerations when designing such systems include resolution, readouts, the specific (micro)environment of cells or tissues, and (epi)genetic relevance. Interactions at different biological levels are of high importance, such as multitissue and multiorganism, where, for example, immune cells and/or paracrine signals, and microbiota, respectively, are key mediators.

Firstly, to obtain a robust model in $\mathrm{OoC}$, it is crucial to have well-characterized cell sources. Selecting the appropriate cells is often based on their availability from commercial sources or primary donors. Tissue biopsies and primary cells, being derived from adult tissues/patient material, reflect a mature phenotype, which can more closely represent the variance within a clinical population [Wnorowski et al., 2019]. To facilitate the access to cell sources, such as iPSCs and organoids, biobanked material is an interesting option [Peck et al., 2020]. In fact, examples of strategic collaborations between lead OoC industrial players, such as MIMETAS, and academic-based organizations, namely Hubrecht Organoid Technologies (organoid biobank), are already in place to facilitate the market translation of organoid-on-chip models. Indeed, human-derived iPSCs are considered a more suitable approach to circumvent the latent limited availability and/ or variability of primary adult cells. iPSCs display a variety of compelling advantages, including: (a) an almost unlimited source of cells; and, (b) generation of isogenic cell lines [Ramme et al., 2018]. Nevertheless, as already described above, the phenotype of the great majority of the available iPSC-derived differentiated cells is typically sustained in an immature state.

Secondly, linking multiple OoC into multiorgan systems requires consideration of several aspects, such as: (a) physiologically relevant order of connected units and their scaling; (b) modelling fluid flow through the system to provide each tissue with the appropriate shear stress; (c) maintenance of sterility when connecting units or during readouts; and, (d) establishment of a compatible shared medium [Wikswo et al., 2013a; Allwardt et al., 2020]. Various scaling strategies have been proposed, including allosteric, functional, and histological sections. Each of these strategies has its own merit and trade-offs, which are reviewed in more details elsewhere [Wikswo et al., 2013b]. Functional coupling indeed involves the perfusion of shared media through the multiple units, which, in turn, will model sequential multiorgan interaction via, for example, vasculature-like channels, in a physiologically relevant fashion [Vernetti et al., 2017; Herland et al., 2020]. In fact, multiorgan compatible cell culture medium is frequently based on mixes of organ-specific culture media [Chang et al., 2017]. However, as the number of linked systems increases, the success of the medium mixing approach could decrease, exposing the organ/tissue units to suboptimal culture conditions, which can detrimentally impact the function and, thereby, the physiological relevance of the whole system. Alternatively, by varying the surface chemistry of the scaffolds on which cells are cultured, the distinct tissues or organs may be provided with specific culture requirements, independent of the source of the shared medium [Oleaga et al., 2016, 2019].

\section{Facilitating the Commercialization and Use of $\mathrm{OoC}$}

For worldwide acceptance and utilization of OoC platforms in a commercial setting, the most important criteria to consider include: (1) reproducibility; (2) ease of scale-up; (3) increased throughput; (4) automation; and, (5) technical standardization of the platforms. Moreover, validating these $\mathrm{OoC}$ platforms and having them adopted by regulatory agencies as valid and representative preclinical models, remains the biggest hurdle facing the widespread implementation of OoC by pharmaceutical companies, for example. In fact, independent qualification laboratories, such as the US National Center for Advancing Translational Sciences and the European Union Ref- 

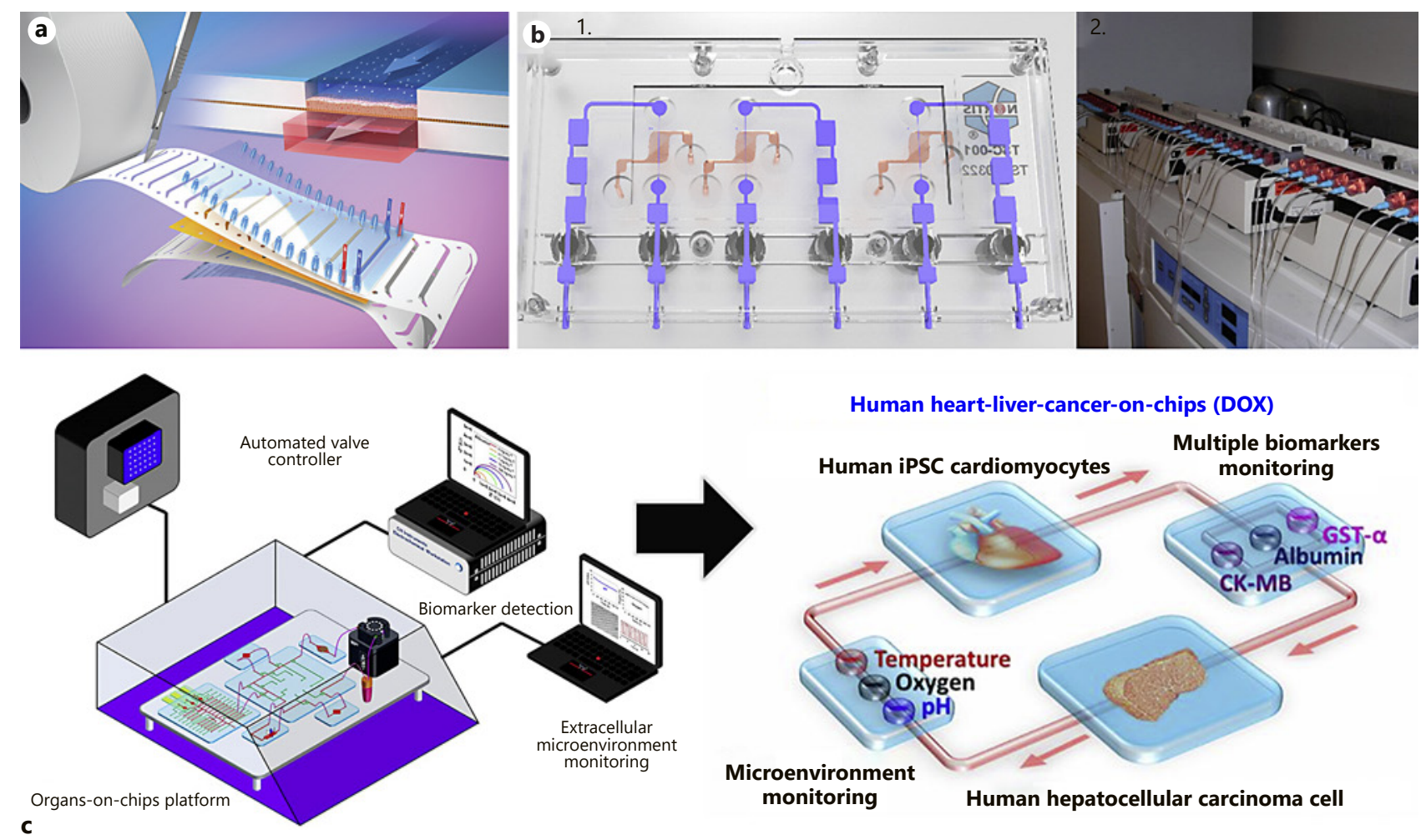

Fig. 4. Examples of OoC systems re-engineered to enable highthroughput and automation. a A barrier-on-chip system fabricated based on double-sided adhesive tape, which renders its industrial manufacture feasible at very low costs per unit. Reproduced from Winkler et al. [2020] with permission from the Royal Society of Chemistry. b 1 . Kidney OoC system, with the perfusion and environmental control system compressed to meet space flight limitations; 2. Conventional laboratory incubator-based and syringe pump-based perfusion system for forty distinct channel chips. The modules are self-contained, housing motors that drive syringe

erence Laboratory for Alternatives to Animal Testing, have been focusing on implementing standardization regulations or guidelines, to guarantee the reproducibility of assays and readouts to, in due course, assure constant effective, reproducible, reliable, and automated OoC systems [Low et al., 2021b].

\section{Scaling up Manufacturing}

Most early OoC designs remain limited by the availability of both manufacturing material and equipment, which compromised scaling up production to an industrially relevant scale. Therefore, academic laboratories are currently establishing shared or "universal" standard op- pump pistons, with full temperature, $\mathrm{CO}_{2}$, and humidity control. Reproduced from Yeung et al., [2020], with permission from Wiley Periodicals Inc. c Schematic representation of an integrated automated multi-OoC, combined with a sensing platform, within an in-house designed benchtop incubator. This heart-liver-canceron-chip system features an automated pneumatic valve controller, electronics for operating physical sensors, potentio-stat for measuring electrochemical signals, and programed command integration, which allows automated in-line toxicity sensing. Reproduced with permission from Zhang et al. [2017b]. erating procedures or setups to assure quality and easy translation towards other manufacturers or users. To accelerate scaling up the manufacture of $\mathrm{OoC}$, partnering between academy and manufacturing companies is dearly needed, as most academic laboratories are not equipped for mass production. Currently, most OoC being developed and applied remain at a relatively low-throughput scale, typically with only few replicates per condition. Consequently, the use of OoC at early stages of drug discovery remains cost- and time-restrictive for stakeholders such as pharmaceutical companies. Currently, examples of commercially available high-throughput OoC include gravity-driven passive flow to supply cells with 
nutrients in either well-plate [Petrosyan et al., 2019; van Duinen et al., 2019] or tape-based formats [Winkler et al., 2020]. In the latter, a simple, low-cost barrier-on-chip successfully modelled intestinal barrier function, which was highly compatible with upscaling (Fig. 4a). An example focused on miniaturization has been reported recently, describing a 3D kidney OoC system, where both perfusion and an environmental control system were compressed to meet space flight limitations (Fig. 4b 1.) [Yeung et al., 2020]. In this condensed OoC platform, all modules are self-contained to house a laboratory incubator-based and syringe pump-based perfusion system for forty chips (Fig. $4 \mathrm{~b}$ 2.). Thus, there is an urgent need to create more automated and miniaturized OoC systems to realize high-throughput, standardized, and easy-to-use platforms.

\section{Automation and Validation}

Commercialization of $\mathrm{OoC}$ based on automation, high-throughput, and standardized quality control will not only contribute to increased cost-effectiveness, but also hasten the wide utilization of these advanced platforms by stakeholders [Whitesides, 2006; Ertl et al., 2014]. The automation path includes: mechanized liquid handling (e.g., microfluidic pumps, microvalves, robotic systems), programmable actuation (e.g., mechanical, electrical), computerized imaging (e.g., monitoring cell proliferation, mobility, and differentiation), embedded sensing (e.g., metabolites, $\mathrm{O}_{2}, \mathrm{pH}$ ) and biomarker analysis (e.g., secreted proteins, activation of specific cellular pathways, membrane receptors, etc.). This level of automation will undoubtedly result in: (1) higher precision and quality control, due to reduction of handling-derived human error; (2) increased reproducibility and compliance with specifications, required by regulating agencies; and, (3) reduction of costs, reflected by the decrease demand of cells, reagents, and human handling time. As a representative example, the automation of eight vascularized $\mathrm{OoC}$ has been successfully validated for long-term cell culture, achieved by programed unit sampling and medium replenishment using a robotic "interrogator," which also included automated image collection of the different units [Novak et al., 2020] (Fig. 3a). Additionally, a system that can work in an automated and continuous manner for a long period of time, through the integration of various biomedical sensors in a multiorgan-chip, has been recently developed (Fig. 4c) [Zhang et al., 2017a]. Besides incorporating an optical function to follow cell morphology and performance, this platform also integrated automatically operating sensing units, including electrochem-

Cells Tissues Organs on-Chip ical immunobiosensors, to detect specific biomarkers, and optical biosensors, to monitor microenvironmental parameters, in real-time.

Obviously, validation of $\mathrm{OoC}$ is essential for commercialization. Validation not purely comprises reproducibility, but also includes in vivo human significance or predictably power. In most of the OoC platforms described above, there was a strong concordance between OoC derived and pre-acquired human clinical data (benchmark comparison), displaying the power of $\mathrm{OoC}$ for preclinical drug development, for example. However, in case such human-derived data are unavailable, comparison to relevant, well-established animal models can be considered an acceptable alternative. As a representative example, the first publication aimed at independently validating various kidney proximal tubule models was recently published [Sakolish et al., 2018], paving the way for many more essential validation studies. Finally, the generation of data in collaboration with established industrial partners, and/or in collaboration with regulatory agencies, would be a tremendous step forward on OoC validation and wide implementation. In this context, it is noteworthy to mention a few companies, namely Emulate, TissUse, and Mimetas, which have been leading the way for widespread adoption of OoC via their collaboration with the FDA and major pharmaceutical partners.

\section{Outlook and Final Remarks}

OoC hold the promise of achieving drug development in a more efficient and cost-effective manner, accommodating for personalized therapy development. OoC are a superb tool for the development of personalized medicine and for knowledge expansion over human diseases. Moreover, this advanced platform will likely contribute to the long-awaited replacement, reduction, and refinement of laboratory animal use. Yet despite facing grand expectations, this technology is still at early development stages and, to reach its full potential, coordinated efforts between academia, industry, and regulatory entities are fundamental to realizing its benefit for society, both clinically and scientifically, where the visible effects will be translated into improving patients' lives.

To bring this exciting technology forward, upscaling, standardization, and validation are fundamental, as well as widely recognized to be urgent. High-throughput of OoC can be achieved by the simplification of design and manufacture, for example, rendering OoC platforms cheap and fast to fabricate, in a controlled and standard- 
ized fashion. Such balance between simplification and system complexity would be ideal for initial stages of drug screening, including drug target identification and hit validation. Multi-OoC systems typically include interconnections between organs or tissues, via shared vascularization and/or innervation, to better mimic systemiclike interactions. This approach, although complex to coordinate and validate, has been proven to be successful by many research groups. This multi-OoC strategy is expected to provide new insights into more intricate systemiclike performance, replicating complex tissue-tissue or organ-organ interactions, which, so far, have only been modelled in vivo. Multi-OoC technology is highly relevant for the later stages of drug screening, more focused on toxicity and efficacy studies, determining the side effects of drugs and patient group treatment stratification, for example.

The foreseen next steps of OoC development may be the implementation of multi-diseases-on-chip, addressing the interactions between diseases or symptoms, which affect several organs, to help determining causality, correlations, or even disentangling disease triggers. This new avenue may also contribute to better defining disease progression profiles and improve the prediction of clinical symptoms or prevention of disease onset at early stages of development. The co-development of OoC with other fields, such as bioimaging (e.g., bioluminescence), will likely follow the routes of label-free, less invasive, and more simple online analysis of the on-chip whole tissues/ organs, as well as at the cellular level. Novel detection and analysis possibilities will help to more accurately study cellular responses and interactions of tissues or organs, possibly even over longer culture timeframes, to better detect the long-term performance and predictability of treatments or disease progression profiles. In the near future of $\mathrm{OoC}$, a rise of predictive power, integrative approaches, automation, and platform mainstreaming is foreseen, to ultimately become an accessible advanced tool from which pharmaceutical research, (bio)medical sciences, and patients can directly benefit.

\section{Conflict of Interest Statement}

The authors have no conflicts of interest to declare.

\section{Funding Sources}

This work is part of the research program Incentive Grants for Women in STEM with project No. 18741, which is (partly) financed by the Dutch Research Council (NWO). This work is also part of the research program ENW Open Competition - KLEIN, with project No. OCENW.KLEIN.352, financed by the NWO.

\section{Author Contributions}

L.S.M.T. proposed the outline of the work; H.M.M.A.M.A. and L.S.M.T. wrote and revised the review; H.M.M.A.M.A. and L.S.M.T. prepared the figures.

\section{References}

Achberger K, Probst C, Haderspeck J, Bolz S, Rogal J, Chuchuy J, et al. Merging organoid and organ-on-a-chip technology to generate complex multi-layer tissue models in a human retina-on-a-chip platform. Elife. 2019;8:e46188.

Allwardt V, Ainscough AJ, Viswanathan P, Sherrod SD, McLean JA, Haddrick M, et al. Translational roadmap for the organs-on-a-chip industry toward broad adoption. Bioengineering. 2020;7(3):112.

An F, Qu Y, Luo Y, Fang N, Liu Y, Gao Z, et al. A laminated microfluidic device for comprehensive preclinical testing in the drug ADME process. Sci Rep. 2016;6(1):25022.

Artegiani B, Hendriks D, Beumer J, Kok R, Zheng $\mathrm{X}$, Joore I, et al. Fast and efficient generation of knock-in human organoids using homology-independent CRISPR-Cas9 precision genome editing. Nat Cell Biol. 2020 Mar;22(3): $321-31$.
Baert Y, Ruetschle I, Cools W, Oehme A, Lorenz A, Marx U, et al. A multi-organ-chip co-culture of liver and testis equivalents: a first step toward a systemic male reprotoxicity model. Hum Reprod. 2020;35(5):1029-44.

Bahmaee H, Owen R, Boyle L, Perrault CM, Garcia-Granada AA, Reilly GC, et al. Design and evaluation of an osteogenesis-on-a-chip microfluidic device incorporating 3D cell culture. Front Bioeng Biotechnol. 2020;8(1042): 557111.

Baker BM, Trappmann B, Stapleton SC, Toro E, Chen CS. Microfluidics embedded within extracellular matrix to define vascular architectures and pattern diffusive gradients. Lab Chip. 2013;13(16):3246-52.

Baker LA, Tiriac H, Clevers H, DAJTic T. Modeling pancreatic cancer with organoids. Trends Cancer. 2016;2(4):176-90.
Barata D, Provaggi E, van Blitterswijk C, Habibovic P. Development of a microfluidic platform integrating high-resolution microstructured biomaterials to study cell-material interactions. Lab Chip. 2017;17(23):4134-47.

Barata D, van Blitterswijk C, Habibovic P. Highthroughput screening approaches and combinatorial development of biomaterials using microfluidics. Acta Biomater. 2016;34:1-20.

Benam KH, Novak R, Nawroth J, Hirano-Kobayashi M, Ferrante TC, Choe Y, et al. Matched-comparative modeling of normal and diseased human airway responses using a microengineered breathing lung chip. Cell Syst. 2016;3(5):456-e4.

Bhise NS, Ribas J, Manoharan V, Zhang YS, Polini A, Massa S, et al. Organ-on-a-chip platforms for studying drug delivery systems. J Control Release. 2014;190:82-93. 
Brooks JC, Judd RL, Easley CJ. Culture and sampling of primary adipose tissue in practical microfluidic systems. Methods Mol Biol. 2017;1566:185-201.

Brown DD, Dabbs DJ, Lee AV, McGuire KP, Ahrendt GM, Bhargava R, et al. Developing in vitro models of human ductal carcinoma in situ from primary tissue explants. Breast Cancer Res Treat. 2015;153(2):311-21.

Bush PG, Hodkinson PD, Hamilton GL, Hall AC. Viability and volume of in situ bovine articular chondrocytes-changes following a single impact and effects of medium osmolarity. Osteoarthritis Cartilage. 2005;13(1):54-65.

Carvalho MR, Barata D, Teixeira LM, Giselbrecht S, Reis RL, Oliveira JM, et al. Colorectal tumor-on-a-chip system: a 3D tool for precision onco-nanomedicine. Sci Adv. 2019;5(5): eaaw1317.

Chang SY, Weber EJ, Sidorenko VS, Chapron A, Yeung CK, Gao C, et al. Human liver-kidney model elucidates the mechanisms of aristolochic acid nephrotoxicity. JCI Insight. 2017; 2(22):e95978.

Cochrane A, Albers HJ, Passier R, Mummery CL, van den Berg A, Orlova VV, et al. Advanced in vitro models of vascular biology: human induced pluripotent stem cells and organ-onchip technology. Adv Drug Deliv Rev. 2019; 140:68-77.

Convery N, Gadegaard NJM. 30 years of microfluidics. Micro Nano Eng. 2019;2:76-91.

Dekkers JF, Wiegerinck CL, De Jonge HR, Bronsveld I, Janssens HM, De Winter-de Groot $\mathrm{KM}$, et al. A functional CFTR assay using primary cystic fibrosis intestinal organoids. Nat Med. 2013;19(7):939-45.

Domachuk P, Tsioris K, Omenetto FG, Kaplan DL. Bio-microfluidics: biomaterials and biomimetic designs. Adv Mater. 2010;22(2):24960.

Driehuis E, Clevers H. CRISPR/Cas 9 genome editing and its applications in organoids. Am J Physiol Gastrointest Liver Physiol. 2017; 312(3):G257-G65.

Du G, Fang Q, den Toonder JMJ. Microfluidics for cell-based high throughput screening platforms - a review. Anal Chim Acta. 2016;903: 36-50.

Duval K, Grover H, Han L-H, Mou Y, Pegoraro AF, Fredberg J, et al. Modeling physiological events in $2 \mathrm{D}$ vs. $3 \mathrm{D}$ cell culture. Physiology. 2017;32(4):266-77.

Edington CD, Chen WLK, Geishecker E, Kassis T, Soenksen LR, Bhushan BM, et al. Interconnected microphysiological systems for quantitative biology and pharmacology studies. Sci Rep. 2018;8(1):4530.

Ertl P, Sticker D, Charwat V, Kasper C, Lepperdinger G. Lab-on-a-chip technologies for stem cell analysis. Trends Biotechnol. 2014; 32(5):245-53.

Everitt JI. The future of preclinical animal models in pharmaceutical discovery and development: a need to bring in cerebro to the in vivo discussions. Toxicol Pathol. 2015 Jan;43(1): 70-7.
Gao D, Li H, Wang N, Lin JM. Evaluation of the absorption of methotrexate on cells and its cytotoxicity assay by using an integrated microfluidic device coupled to a mass spectrometer. Anal Chem. 2012;84(21):9230-7.

Geraili A, Jafari P, Hassani MS, Araghi BH, Mohammadi MH, Ghafari AM, et al. Controlling differentiation of stem cells for developing personalized organ-on-chip platforms. Adv Healthc Mater. 2018;7(2):1700426.

Gnecco JS, Ding T, Smith C, Lu J, Bruner-Tran KL, Osteen KG. Hemodynamic forces enhance decidualization via endothelial-derived prostaglandin E2 and prostacyclin in a microfluidic model of the human endometrium. Hum Reprod. 2019;34(4):702-14.

Gottardi R. Load-induced osteoarthritis on a chip. Nat Biomed Eng. 2019;3(7):502-3.

Halldorsson S, Lucumi E, Gómez-Sjöberg R, Fleming RMT. Advantages and challenges of microfluidic cell culture in polydimethylsiloxane devices. Biosens Bioelectron. 2015;63: 218-31.

Hassell BA, Goyal G, Lee E, Sontheimer-Phelps A, Levy O, Chen CS, et al. Human organ chip models recapitulate orthotopic lung cancer growth, therapeutic responses, and tumor dormancy in vitro. Cell Rep. 2017;21(2):50816.

Herland A, Maoz BM, Das D, Somayaji MR, Prantil-Baun R, Novak R, et al. Quantitative prediction of human pharmacokinetic responses to drugs via fluidically coupled vascularized organ chips. Nat Biomed Eng. 2020;4(4):42136.

Huh D, Matthews BD, Mammoto A, MontoyaZavala M, Hsin HY, Ingber DE. Reconstituting organ-level lung functions on a chip. Science. 2010a;328(5986):1662-8.

Huh D, Matthews BD, Mammoto A, MontoyaZavala M, Hsin HY, Ingber DE. Reconstituting organ-level lung functions on a chip. Science. 2010b;328(5986):1662-8.

Huh D, Hamilton GA, Ingber DE. From 3D cell culture to organs-on-chips. Trends Cell Biol. 2011a;21(12):745-54.

Huh D, Hamilton GA, Ingber DE. From 3D cell culture to organs-on-chips. Trends Cell Biol. 2011b;21(12):745-54.

Jeong SY, Lee JH, Shin Y, Chung S, Kuh HJ. Coculture of tumor spheroids and fibroblasts in a collagen matrix-incorporated microfluidic chip mimics reciprocal activation in solid tumor microenvironment. PLoS One. 2016; 11(7):e0159013.

Johnson CI, Argyle DJ, Clements DN. In vitro models for the study of osteoarthritis. Vet J. 2016;209:40-9.

Kaarj K, Yoon J-YJM. Methods of delivering mechanical stimuli to organ-on-a-chip. Micromachines. 2019;10(10):700.

Kankala R, Xu X-M, Liu C-G, Chen A-Z, Wang S-B. 3D-printing of microfibrous porous scaffolds based on hybrid approaches for bone tissue engineering. Polymers. 2018;10(7):807.
Kim HJ, Huh D, Hamilton G, Ingber DE. Human gut-on-a-chip inhabited by microbial flora that experiences intestinal peristalsis-like motions and flow. Lab Chip. 2012;12(12):216574.

Knowlton S, Yenilmez B, SJTib T. Towards single-step biofabrication of organs on a chip via 3D printing. Trends Biotechnol. 2016;34(9): 685-8.

Kopper O, de Witte CJ, Lõhmussaar K, Valle-Inclan JE, Hami N, Kester L, et al. An organoid platform for ovarian cancer captures intraand interpatient heterogeneity. Nat Med. 2019;25(5):838-49.

Lancaster MA, Knoblich JA. Organogenesis in a dish: modeling development and disease using organoid technologies. Science. 2014; 345(6194): 1247125.

Langerak N, Ahmed HMM, Li Y, Middel IR, Eslami Amirabadi $\mathrm{H}$, Malda J, et al. A theoretical and experimental study to optimize cell differentiation in a novel intestinal chip. Front Bioeng Biotechnol. 2020;8(763):763.

Lee J-H, Kim S-K, Khawar IA, Jeong S-Y, Chung S, Kuh H-J, et al. Microfluidic co-culture of pancreatic tumor spheroids with stellate cells as a novel 3D model for investigation of stroma-mediated cell motility and drug resistance. J Exp Clin Cancer Res. 2018;37(1):412.

Lee SY, Sung JH. Gut-liver on a chip toward an in vitro model of hepatic steatosis. Biotechnol Bioeng. 2018;115(11):2817-27.

Liu W, Song J, Du X, Zhou Y, Li Y, Li R, et al. AKR1B10 (Aldo-keto reductase family 1 B10) promotes brain metastasis of lung cancer cells in a multi-organ microfluidic chip model. Acta Biomater. 2019;91:195-208.

Lochovsky C, Yasotharan S, Günther AJLC. Bubbles no more: in-plane trapping and removal of bubbles in microfluidic devices. Lab Chip. 2012;12(3):595-601.

Low LA, Mummery C, Berridge BR, Austin CP, Tagle DA. Organs-on-chips: into the next decade. Nat Rev Drug Discov. 2021a;20(5):34561.

Low LA, Mummery C, Berridge BR, Austin CP, Tagle DA. Organs-on-chips: Into the next decade. Nat Rev Drug Discov. 2021b;20(5):34561.

Low LA, Sutherland M, Lumelsky N, Selimovic S, Lundberg MS, Tagle DA. Organs-on-a-chip. Adv Exp Med Biol. 2020;1230:27-42.

Mandrycky C, Wang Z, Kim K, Kim D-HJB. 3D bioprinting for engineering complex tissues. Biotechnol Adv. 2016;34(4):422-34.

Maoz BM, Herland A, Henry OYF, Leineweber WD, Yadid M, Doyle J, et al. Organs-on-chips with combined multi-electrode array and transepithelial electrical resistance measurement capabilities. Lab Chip. 2017;17(13): 2294-302.

Marx U. Trends in cell culture technology. In: Balls M, Combes RD, Bhogal N, editors. New technologies for toxicity testing. New York: Springer US; 2012. p. 26-46 
Maschmeyer I, Lorenz AK, Schimek K, Hasenberg T, Ramme AP, Hübner J, et al. A fourorgan-chip for interconnected long-term coculture of human intestine, liver, skin and kidney equivalents. Lab Chip. 2015a;15(12): 2688-99.

Maschmeyer I, Lorenz AK, Schimek K, Hasenberg T, Ramme AP, Hübner J, et al. A fourorgan-chip for interconnected long-term coculture of human intestine, liver, skin and kidney equivalents. Lab Chip. 2015b;15(12): 2688-99.

Matano M, Date S, Shimokawa M, Takano A, Fujii $\mathrm{M}$, Ohta $\mathrm{Y}$, et al. Modeling colorectal cancer using CRISPR-Cas9-mediated engineering of human intestinal organoids. Nat Med. 2015;21(3):256-62.

Mead BE, Karp JM. All models are wrong, but some organoids may be useful. Genome Biol. 2019;20(1):66.

Middelkamp H, van der Meer A, Hummel M, Stamatialis D, Mummery C, Passier R, et al. Organs-on-chips in drug development: the importance of involving stakeholders in early health technology assessment. Appl In Vitro Toxicol. 2016;2:10.1089/aivt.2015.0029.

Miller CP, Tsuchida C, Zheng Y, Himmelfarb J, Akilesh SJN. A 3D human renal cell carcinoma-on-a-chip for the study of tumor angiogenesis. Neoplasia. 2018;20(6):610-20.

Moradi E, Jalili-Firoozinezhad S, Solati-Hashin M. Microfluidic organ-on-a-chip models of human liver tissue. Acta Biomater. 2020;116: 67-83.

Moroni L, Burdick JA, Highley C, Lee SJ, Morimoto Y, Takeuchi S, et al. Biofabrication strategies for $3 \mathrm{D}$ in vitro models and regenerative medicine. Nat Rev Mater. 2018;3(5):21-37.

Nawroth JC, Barrile R, Conegliano D, van Riet S, Hiemstra PS, Villenave R. Stem cell-based lung-on-chips: the best of both worlds? Adv Drug Deliv Rev. 2019;140:12-32.

Nouri-Goushki M, Sharma A, Sasso L, Zhang S, Van der Eerden BCJ, Staufer U, et al. Submicron patterns-on-a-chip: fabrication of a microfluidic device incorporating 3D printed surface ornaments. ACS Biomater Sci Eng. 2019;5(11):6127-36.

Novak R, Ingram M, Marquez S, Das D, Delahanty A, Herland A, et al. Robotic fluidic coupling and interrogation of multiple vascularized organ chips. Nat Biomed Eng. 2020;4(4): 407-20.

Occhetta P, Mainardi A, Votta E, Vallmajo-Martin Q, Ehrbar M, Martin I, et al. Hyperphysiological compression of articular cartilage induces an osteoarthritic phenotype in a cartilage-on-a-chip model. Nat Biomed Eng. 2019 Jul;3(7):545-57.

Oleaga C, Bernabini C, Smith AST, Srinivasan B, Jackson M, McLamb W, et al. Multi-organ toxicity demonstration in a functional human in vitro system composed of four organs. Sci Rep. 2016;6(1):20030.
Oleaga C, Riu A, Rothemund S, Lavado A, McAleer CW, Long CJ, et al. Investigation of the effect of hepatic metabolism on off-target cardiotoxicity in a multi-organ human-on-achip system. Biomaterials. 2018;182:176-90.

Oleaga C, Lavado A, Riu A, Rothemund S, Carmona-Moran CA, Persaud K, et al. Long-term electrical and mechanical function monitoring of a human-on-a-chip system. 2019;29(8): 1805792.

Pampaloni F, Reynaud EG, Stelzer EHK. The third dimension bridges the gap between cell culture and live tissue. Nat Rev Mol Cell Biol. 2007;8(10):839-45.

Park JY, Yoo SJ, Hwang CM, Lee S-H. Simultaneous generation of chemical concentration and mechanical shear stress gradients using microfluidic osmotic flow comparable to interstitial flow. Lab Chip. 2009;9(15):2194-202.

Park S-H, Sim WY, Min B-H, Yang SS, Khademhosseini A, Kaplan DL. Chip-based comparison of the osteogenesis of human bone marrow- and adipose tissue-derived mesenchymal stem cells under mechanical stimulation. PLoS One. 2012;7(9):e46689.

Park JY, Jang J, Kang H-W. 3D Bioprinting and its application to organ-on-a-chip. Microelectron Eng. 2018;200:1-11.

Park SE, Georgescu A, Huh D. Organoids-on-achip. Science. 2019;364(6444):960-5.

Peck RW, Hinojosa CD, Hamilton GA. Organson-chips in clinical pharmacology: putting the patient into the center of treatment selection and drug development. Clin Pharmacol Ther. 2020;107(1):181-5.

Petrosyan A, Cravedi P, Villani V, Angeletti A, Manrique J, Renieri A, et al. A glomeruluson-a-chip to recapitulate the human glomerular filtration barrier. Nat Commun. 2019; 10(1):3656.

Pound P, Ritskes-Hoitinga M. Is it possible to overcome issues of external validity in preclinical animal research? Why most animal models are bound to fail. J Transl Med. 2018; 16(1):304.

Puca L, Bareja R, Prandi D, Shaw R, Benelli M, Karthaus WR, et al. Patient derived organoids to model rare prostate cancer phenotypes. Nat Commun. 2018;9(1):2404-10

Ramme AP, Koenig L, Hasenberg T, Schwenk C, Magauer C, Faust D, et al. Towards an autologous iPSC-derived patient-on-a-chip. bioRxiv. 2018. doi: https://doi.org/10.1101/376970.

Regehr KJ, Domenech M, Koepsel JT, Carver KC, Ellison-Zelski SJ, Murphy WL, et al. Biological implications of polydimethylsiloxanebased microfluidic cell culture. Lab Chip. 2009;9(15):2132-9.

Ronaldson-Bouchard K, Vunjak-Novakovic G. Organs-on-a-chip: a fast track for engineered human tissues in drug development. Cell Stem Cell. 2018;22(3):310-24.

Rothbauer M, Zirath H, Ertl P. Recent advances in microfluidic technologies for cell-to-cell interaction studies. Lab Chip. 2018;18(2): 249-70.
Saeidnia S, Manayi A, Abdollahi M. From in vitro experiments to in vivo and clinical studies: pros and cons. Curr Drug Discov Technol. 2015;12(4):218-24.

Sakolish C, Weber EJ, Kelly EJ, Himmelfarb J, Mouneimne R, Grimm FA, et al. Technology transfer of the microphysiological systems: a case study of the human proximal tubule tissue chip. Sci Rep. 2018;8(1):14882.

Sateesh J, Guha K, Dutta A, Sengupta P, Srinivasa Rao K. Regenerating re-absorption function of proximal convoluted tubule using microfluidics for kidney-on-chip applications. SN Appl Sci. 2019;2(1):39.

Sato T, Van Es JH, Snippert HJ, Stange DE, Vries RG, Van Den Born M, et al. Paneth cells constitute the niche for Lgr 5 stem cells in intestinal crypts. Nature. 2011;469(7330):415-8.

Shim K-Y, Lee D, Han J, Nguyen N-T, Park S, Sung JH. Microfluidic gut-on-a-chip with three-dimensional villi structure. Biomed Microdevices. 2017;19(2):37.

Stone HA, Stroock AD, Ajdari AJARFM. Engineering flows in small devices: microfluidics toward a lab-on-a-chip. Ann Rev Fluid Mech. 2004;36:381-411.

Sung JH, Srinivasan B, Esch MB, McLamb WT, Bernabini C, Shuler ML, et al. Using physiologically-based pharmacokinetic-guided "body-on-a-chip" systems to predict mammalian response to drug and chemical exposure. Exp Biol Med. 2014a;239(9):1225-39.

Sung JH, Srinivasan B, Esch MB, McLamb WT, Bernabini C, Shuler ML, et al. Using physiologically-based pharmacokinetic-guided "body-on-a-chip" systems to predict mammalian response to drug and chemical exposure. Exp Biol Med. 2014b;239(9):1225-39.

Suvarnapathaki S, Wu X, Lantigua D, Nguyen MA, Camci-Unal G. Breathing life into engineered tissues using oxygen-releasing biomaterials. NPG Asia Mater. 2019;11(1):65.

Takasato M, Pei XE, Chiu HS, Maier B, Baillie GJ, Ferguson C, et al. Kidney organoids from human iPS cells contain multiple lineages and model human nephrogenesis. Nature. 2015; 526(7574):564-8.

Takeda H, Kataoka S, Nakayama M, Ali MAE, Oshima H, Yamamoto D, et al. CRISPR-Cas9mediated gene knockout in intestinal tumor organoids provides functional validation for colorectal cancer driver genes. Proc Natl Acad Sci USA. 2019;116(31):15635-44.

Ting L, Feghhi S, Karchin A, Tooley W, White NJ, Sniadecki N. Clot-on-a-chip: a microfluidic device to study platelet aggregation and contractility under shear. Blood. 2013;122(21): 2363-3.

Toepke MW, Beebe DJJLC. PDMS absorption of small molecules and consequences in microfluidic applications. Lab Chip. 2006;6(12): 1484-6. 
Trapecar M, Communal C, Velazquez J, Maass CA, Huang Y-J, Schneider K, et al. Gut-Liver physiomimetics reveal paradoxical modulation of IBD-related inflammation by short-chain fatty acids. Cell Syst. 2020;10(3):223-9.e9.

van Duinen V, Stam W, Borgdorff V, Reijerkerk A, Orlova V, Vulto P, et al. Standardized and scalable assay to study perfused $3 \mathrm{~d}$ angiogenic sprouting of ipsc-derived endothelial cells in vitro. J Vis Exp. 2019;6(153):59678.

Vernetti L, Gough A, Baetz N, Blutt S, Broughman JR, Brown JA, et al. Functional coupling of human microphysiology systems: intestine, liver, kidney proximal tubule, bloodbrain barrier and skeletal muscle. Sci Rep. 2017;7:42296

Waheed S, Cabot JM, Macdonald NP, Lewis T, Guijt RM, Paull B, et al. 3D printed microfluidic devices: enablers and barriers. Lab Chip. 2016;16(11):1993-2013.

Wang X, Liu Z, Pang Y. Concentration gradient generation methods based on microfluidic systems. RSC Adv. 2017;7(48):29966-84.

Watson DE, Hunziker R, Wikswo JP. Fitting tissue chips and microphysiological systems into the grand scheme of medicine, biology, pharmacology, and toxicology. Exp Biol Med. 2017;242(16):1559-72.

Whitesides GM. The origins and the future of microfluidics. Nature. 2006;442(7101):368-73.
Wikswo JP, Block FE 3rd, Cliffel DE, Goodwin CR, Marasco CC, Markov DA, et al. Engineering challenges for instrumenting and controlling integrated organ-on-chip systems. IEEE Trans Biomed Eng. 2013a;60(3):682-90.

Wikswo JP, Curtis EL, Eagleton ZE, Evans BC, Kole A, Hofmeister LH, et al. Scaling and systems biology for integrating multiple organson-a-chip. Lab Chip. 2013b;13(18):3496511.

Winkler TE, Feil M, Stronkman EFGJ, Matthiesen I, Herland A. Low-cost microphysiological systems: feasibility study of a tape-based barrier-on-chip for small intestine modeling. Lab Chip. 2020;20(7):1212-26.

Wnorowski A, Yang H, Wu JC. Progress, obstacles, and limitations in the use of stem cells in organ-on-a-chip models. Adv Drug Deliv Rev. 2019;140:3-11.

Xiao S, Coppeta JR, Rogers HB, Isenberg BC, Zhu J, Olalekan SA, et al. A microfluidic culture model of the human reproductive tract and 28-day menstrual cycle. Nat Commun. 2017; $8(1): 14584$.

Yeung CK, Koenig P, Countryman S, Thummel KE, Himmelfarb J, Kelly EJ. Tissue chips in space - challenges and opportunities. Clin Transl Sci. 2020;13(1):8-10.

Yue K, Trujillo-de Santiago G, Alvarez MM, Tamayol A, Annabi N, Khademhosseini A. Synthesis, properties, and biomedical applications of gelatin methacryloyl (GelMA) hydrogels. Biomaterials. 2015;73:254-71.
Zambito G, Gaspar N, Ridwan Y, Hall MP, Shi C, Kirkland TA, et al. Evaluating brightness and spectral properties of click beetle and firefly luciferases using luciferin analogues: identification of preferred pairings of luciferase and substrate for in vivo bioluminescence imaging. Mol Imaging Biol. 2020;22(6):1523-31.

Zhang YS, Arneri A, Bersini S, Shin S-R, Zhu K, Goli-Malekabadi Z, et al. Bioprinting 3D microfibrous scaffolds for engineering endothelialized myocardium and heart-on-a-chip. Biomaterials. 2016;110:45-59.

Zhang YS, Aleman J, Shin SR, Kilic T, Kim D, Mousavi Shaegh SA, et al. Multisensor-integrated organs-on-chips platform for automated and continual in situ monitoring of organoid behaviors. Proc Natl Acad Sci USA. 2017a;114(12):E2293-E302.

Zhang YS, Aleman J, Shin SR, Kilic T, Kim D, Mousavi Shaegh SA, et al. Multisensor-integrated organs-on-chips platform for automated and continual in situ monitoring of organoid behaviors. Proc Natl Acad Sci USA. 2017b;114(12):E2293-E302.

Zhao Y, Rafatian N, Wang EY, Wu Q, Lai BFL, Lu RX, et al. Towards chamber specific heart-ona-chip for drug testing applications. Adv Drug Deliv Rev. 2020;165-6:60-76.

Zhu M, Wang Y, Ferracci G, Zheng J, Cho N-J, Lee BH. Gelatin methacryloyl and its hydrogels with an exceptional degree of controllability and batch-to-batch consistency. Sci Rep. 2019;9(1):6863. 